\title{
Comparison of Efficacy of Intravitreal Aflibercept and Ranibizumab in Treatment-naive Diabetic Macular Edema
}

\author{
(D) Burak Erden¹, (D) Akın Çakır¹, (D) Selim Bölükbaşı1 ${ }^{1}$, (D) Ali Cihat Aslan², (D) Mustafa Nuri Elçioğlu1 \\ 1 istanbul Okmeydanı Training and Research Hospital, Clinic of Ophthalmology, Istanbul, Turkey \\ 2Urfa State Hospital, Clinic of Ophthalmology, Şanlıurfa, Turkey
}

\section{Abstract}

Objective: The purpose of this study was to compare the efficacy of two different intravitreal anti-vascular endothelial growth factor (anti-VEGF) agents in two treatmentnaive and statistically equal cohorts of diabetic macular edema patients.

Methods: In this retrospective study, 81 eyes of 64 treatment-naive diabetic macular edema (DME) patients were enrolled. Patients were divided into two groups and both groups were treated [37 eyes with intravitreal $0.5 \mathrm{mg}$ ranibizumab (IVR) and 44 eyes with intravitreal $2 \mathrm{mg}$ aflibercept (IVA)] with three consecutive injections at intervals of one month. All patients underwent a detailed eye examination including optic coherence tomography and best corrected visual acuity (BCVA; Snellen), biomicroscopy, fundoscopy and applanation tonometry at preoperative, $1^{\text {st }}, 2^{\text {nd }}$ and $3^{\text {rd }}$ month. BCVA values were converted into logarithm of the minimum angle of resolution (IogMAR) for statistical analyses. Data were evaluated with SPSS 25.0.

Results: Mean BCVA (logMAR) increased from $0.58 \pm 0.28$ to $0.43 \pm 0.29,0.39 \pm 0.25$ and $0.32 \pm 0.26(p=0.001, p<0.001, p<0.001)$ in the IVR group and from $0.54 \pm 0.28$ to $0.41 \pm 0.34,0.43 \pm 0.39$ and to $0.32 \pm 0.37$ ( $p=0.004, p=0.023, p<0.001$ ) in the IVA group. Mean central macular thickness (CMT) decreased from $406 \pm 82 \mu \mathrm{m}$ to $345 \pm 65$ $\mu \mathrm{m}$ (15t month), $332 \pm 83 \mu \mathrm{m}$ ( $2^{\text {nd }}$ month) and finally to $303 \pm 60 \mu \mathrm{m}$ ( $3^{\text {rd }}$ month) $(p<0.001)$ in the IVR group and from $415 \pm 88 \mu \mathrm{m}$ to $328 \pm 79 \mu \mathrm{m}, 297 \pm 54 \mu \mathrm{m}$ and finally to $277 \pm 54 \mu \mathrm{m}(p<0.001)$ in the IVA group, respectively. There was no significant difference between the groups in terms of BCVA ( $p>0.05)$. In the subgroup analysis, CMT gain in patients with moderate DME $(C M T \leq 385 \mu \mathrm{m})$ was found significantly better in the IVA group compared to the IVR group $\left(1^{\text {st }}\right.$ month: $36.9 \mathrm{vs}$. $83.6,2^{\text {nd }}$ month: 36.2 vs. $106.3,3^{\text {rd }}$ month: $3^{\text {nd }} 72.7$ vs. $\left.125.1 ; p<0.05\right)$.

Conclusion: Both anti-VEGFs were equally effective in visual outcomes. Compared to ranibizumab, aflibercept has a rapid and superior therapeutic effect in anatomical results, especially in moderate DME cases.

Keywords: Aflibercept, anti-vascular endothelial growth factor, diabetic macular edema, ranibizumab

\section{INTRODUCTION}

Diabetic macular edema (DME) is the most common cause of visual impairment in the diabetic population (1). According to a metaanalysis of 22.896 diabetic patients, the prevalence of centerinvolving DME was 6.81\% (2). Historically, several interventional therapies such as focal/grid laser photocoagulation, intravitreal/ periocular corticosteroids (triamcinolone acetonide etc.) or pars plana vitrectomy have proven to be effective in the treatment of focal or diffuse DME. However, intravitreal injections of antivascular endothelial growth factor (anti-VEGF) drugs with safe and effective profile have been considered as first-line therapy for DME in the last decade. Recently, the results of Protocol T trial of Diabetic Retinopathy Clinical Research Network (DRCR. net) - the most discussed comparison of three anti-VEGF drugs (ranibizumab, aflibercept on-label and bevacizumab off-label) - were published, including the post-hoc analysis in several publications (3,4). In this study, we aimed to compare the efficacy of two on-label anti-VEGFs in two comparable, treatment-naive diabetic edema cohorts under real-life conditions.

\section{METHODS}

This study was conducted at the Istanbul Okmeydanı Training and Research Hospital, Clinic of Ophthalmology. The study was approved by Clinical Research Ethics Committee of Okmeydanı Training and Research Hospital and adhered to the principles of the Declaration of Helsinki. Initially, medical records and 


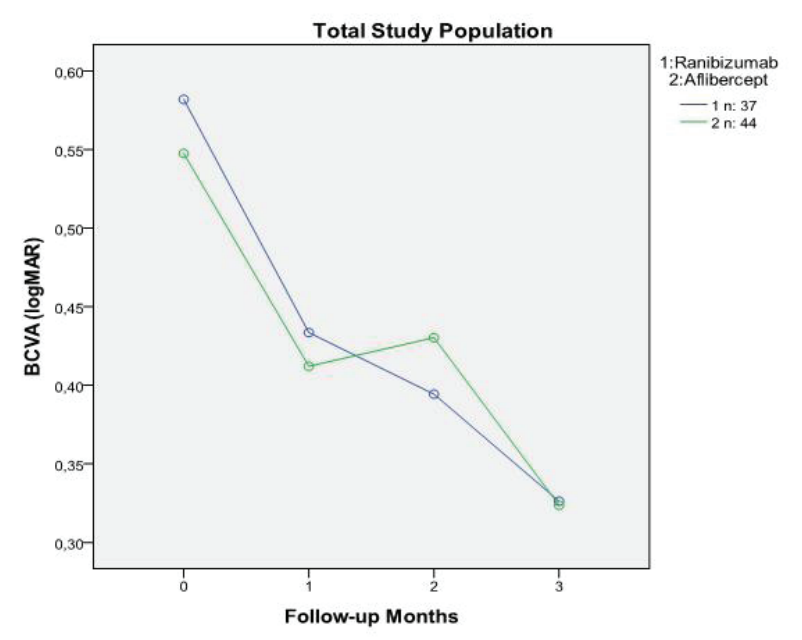

Figure 1. The visual gain comparison in the total study group IogMAR: Logarithm of the minimum angle of resolution, BCVA: Best corrected visual acuity

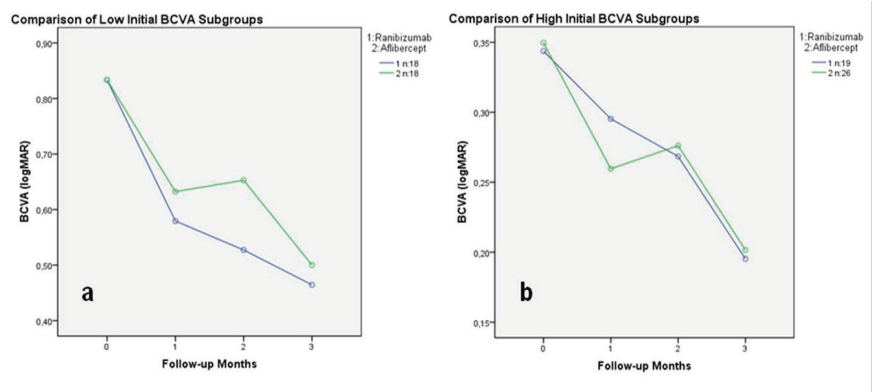

Figure 2. a) Comparison of intravitreal aflibercept and intravitreal ranibizumab groups in visual prognosis in low baseline best corrected visual acuity subgroup b) and in high baseline best corrected visual acuity subgroup

IogMAR: Logarithm of the minimum angle of resolution, BCVA: Best corrected visual acuity

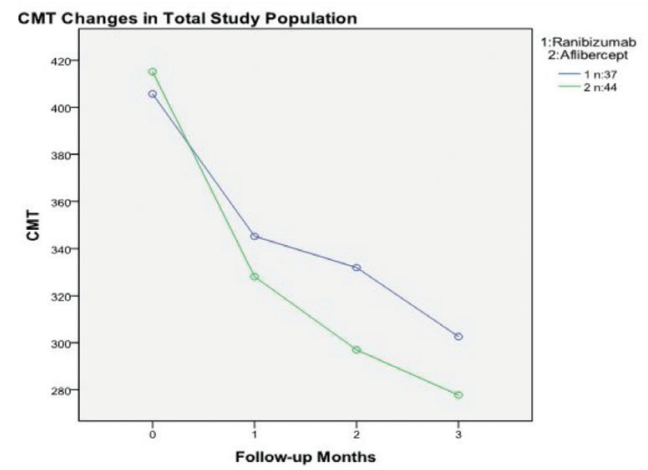

Figure 3. The regression of macular edema in the total study group CMT: Central macular thickness

\section{Anatomical Outcome}

The mean CMT was significantly reduced in the IVR group from $406 \pm 82 \mu \mathrm{m}$ to $345 \pm 65 \mu \mathrm{m}$ (1 $1^{\text {st }}$ month), $332 \pm 83 \mu \mathrm{m}$ ( $2^{\text {nd }}$ month) and finally to $303 \pm 60 \mu \mathrm{m}$ ( $3^{\text {rd }}$ month) $(p<0.001)$, respectively.
In the IVA group, the baseline mean CMT also decreased significantly from $415 \pm 88 \mu \mathrm{m}$ to $328 \pm 79 \mu \mathrm{m}$ ( $1^{\text {st }}$ month), $297 \pm 54 \mu \mathrm{m}\left(2^{\text {nd }}\right.$ month $)$ and finally to $277 \pm 54 \mu \mathrm{m}$ ( $3^{\text {rd }}$ month $)$ $(p<0.001)$, respectively. The intergroup comparison of monthly CMT values showed a significant superiority of IVA group at the $2^{\text {nd }}$ month visit $(p=0.03)$ (Figure 3). Additionally, the anatomical gain comparisons between IVR and IVA groups ( 60 vs. $87 \mu \mathrm{m} ; 73$ vs. $118 \mu \mathrm{m} ; 103$ vs. $137 \mu \mathrm{m}$ ) indicated a general superiority in the IVA group over IVR group, which was statistically significant at $2^{\text {nd }}$ month visit ( $p=0.09 ; p=0.03 ; p=0.07$, respectively). In the subgroup analysis of anatomical evaluation, we divided the total study group into severe and moderate DME subgroups according to the cut-off value (385 $\mu$ m; median value). In severe DME (CMT $>385 \mu \mathrm{m}$; IVR $\mathrm{n}=18$; IVA $\mathrm{n}=22$ ) cases, the mean CMT value in the IVR cohort decreased from $472 \pm 64 \mu \mathrm{m}$ to $371 \pm 81 \mu \mathrm{m}$, to $340 \pm 94 \mu \mathrm{m}$ and finally to $313 \pm 78 \mu \mathrm{m}$, respectively. In the IVA group, the mean CMT decreased from $483 \pm 74 \mu \mathrm{m}$ to $362 \pm 92$ $\mu \mathrm{m}$, to $320 \pm 56 \mu \mathrm{m}$ and finally to $291 \pm 61 \mu \mathrm{m}$, respectively. The intergroup comparison of CMT reduction in this severe DME subgroup revealed no significance $(p=0.42)$ (Figure 4a). On the other hand, the mean CMT in IVR group with moderate DME decreased significantly from $343 \pm 28 \mu \mathrm{m}$ to $321 \pm 32 \mu \mathrm{m}$, to $323 \pm 72 \mu \mathrm{m}$ and finally to $292 \pm 34 \mu \mathrm{m}$ at $3^{\text {rd }}$ month visit $(p<0.01)$. However, in the IVA group, a rapid and greater CMT reduction (from $346 \pm 24 \mu \mathrm{m}$ to $294 \pm 45 \mu \mathrm{m}$, to $273 \pm 41 \mu \mathrm{m}$ and to $264 \pm 45$ $\mu \mathrm{m} ; \mathrm{p}<0.001)$ was observed. The comparison of both anti-VEGF agents in moderate DME cases revealed a statistically significant difference in favor of IVA group during the follow-up ( $p=0.03)$, starting from the $1^{\text {st }}$ month visit (Figure $4 b$ ).

\section{DISCUSSION}

The major cause of visual loss in diabetic population with nonproliferative retinopathy is center-involving DME. The anti-VEGF drugs have dominated clinicians' treatment approach over the
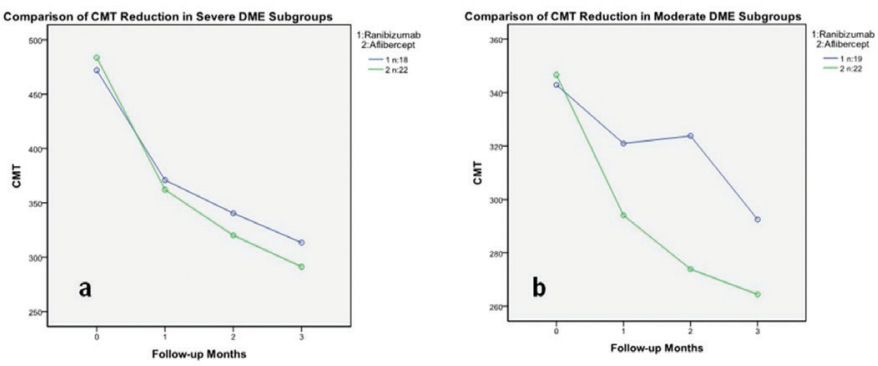

Figure 4. a) The subgroup analyses of central macular thickness reduction in response to intravitreal aflibercept and intravitreal ranibizumab treatments in severe macular edema cases b) and in moderate macular edema cases

CMT: Central macular thickness 
last decade, with promising results and relative safety starting with the off-label use of bevacizumab (5). Following the usage of bevacizumab, two on-label agents, ranibizumab and aflibercept, were introduced into our daily practice with superior results from their representative trials (6,7). In addition, anti-VEGF agents have proved to be superior to conventional therapies. Monotherapy with IVR showed better clinical results than conventional laser photocoagulation (8). Corticosteroids such as dexamethasone has well-known potential risks such as cataract progression and glaucoma, so they have a limited indication in the treatment of DME patients (9). Therefore, clinicians prefer anti-VEGFs as the first-line therapy for this common clinical entity (10).

The on-going debate about which anti-VEGF would be recommended in each individual case has often been dependent on the clinician's experience in daily practice, local administrative regulations of the countries or financial issues. Recently, the comparative clinical trial Protocol T of DRCR.net reported first and second year results $(3,4)$. While the overall visual results of the first year did not reveal any statistical difference between these three anti-VEGFs, aflibercept was significantly superior compared to ranibizumab $(p=0.0003)$ and bevacizumab $(p=0.0001)$ in the lower baseline BCVA $(\leq 20 / 50)$ subgroup (3). Regarding the anatomical results of the whole study population, the greatest decrease in mean CMT was found in the aflibercept group $(169 \pm 139 \mu \mathrm{m}$ vs. $147 \pm 134 \mu \mathrm{m}$ vs. $101 \pm 121 \mu \mathrm{m})$ at the end of the $1^{\text {st }}$ year. However, for the $2^{\text {nd }}$ year results of this trial, the ranibizumab group caught up on the aflibercept group both in visual and anatomical gains (12.8 letters vs. 12.3 letters; $171 \pm 141 \mu \mathrm{m}$ vs. $+149 \pm 141 \mu \mathrm{m})$, both on-label anti-VEGFs remained their superiority over bevacizumab (4). The Protocol T results partially supported the theoretical superiority aflibercept in visual gain, especially in low-vision cases. Therefore, we tried to compare ranibizumab and aflibercept in different baseline BCVA subgroups and aimed to find any differences in a particular clinical situation. Contrary to Protocol T findings in low baseline BCVA subgroup analysis, the IVA group in our study did not differ from the IVR group at any particular visit. Both anti-VEGF groups reached comparable functional endpoints at the final visit, such as the final result of the $2^{\text {nd }}$ year of the Protocol $T$ trial.

The reason for better efficacy of aflibercept over the $1^{\text {st }}$ year results of the Protocol T trial may be due to its broader pharmacological features. In contrast to the antibody-based VEGF binding mechanism of ranibizumab and bevacizumab, aflibercept blocks the specific binding domains of the VEGF receptor (VEGFR)-1 and the VEGFR-2 (11). Aflibercept binds all isoforms of VEGF-A like the other two anti-VEGFs, additionally it also binds VEGF-B and placental growth factor, and the intermediate size of the molecule ( $110 \mathrm{kD}$, compared to $48 \mathrm{kD}$ for ranibizumab and $148 \mathrm{kD}$ for bevacizumab) create a potential monthly intravitreal activity that theoretically exceeds both ranibizumab and bevacizumab (12). This long-lasting effect is also reflected in some practical clinical reports. In their study comparing ranibizumab and aflibercept, Shimizu et al. (13) concluded that visual improvement in DME patients following consecutive intravitreal injections lasted significantly longer in aflibercept group than in ranibizumab group (6 vs. 3 months). In the IVA arm of their report, a subgroup of the patients had previous IVR treatment for DME. They found that IVA treatment did not improve the visual acuity further in previously treated IVR subgroup, but that the mean CMT decreased equally in both subgroups with or without prior IVR history. We believe this finding actually points to the additional anatomical efficacy of aflibercept. In the subgroup analysis of anatomical results of our study, IVA and IVR were found to be comparatively effective in the total study population, except for the $2^{\text {nd }}$ month visit, and in the severe DME subgroup, but IVA proved to be significantly more effective in moderate DME cases. As a general rule, the more severe the macular edema, the more dramatically the CMT will decrease as a response to an effective treatment modality, however, the fact being significantly more effective in reducing the mean CMT of moderate DME cases clearly indicated the superior anatomical efficacy of aflibercept, probably due to its VEGF-trap character. The major limitations of this current study were clearly its retrospective nature and the absence of any randomization. We tried to eliminate the biases arising from its design by reviewing a large number of patient data and enrolling only eligible patients in the IVR and IVA groups to conduct two comparable treatment arms. Unlike representative clinical trials of anti-VEGFs $(14,15)$, where patients get a much higher amount (7-12 times/year) of regular injections, real life based reports such as the Pride Study (16) reveal a much lesser frequency of intravitreal treatments (4 IVR injections/18 months). This fact clearly emphasizes the difference between randomized clinical trials and evidence findings in the real world.

\section{CONCLUSION}

In conclusion, our results demonstrated that ranibizumab and aflibercept were equally effective in visual prognoses of treatment-naive center-involving DME cases. Aflibercept distinguished itself in anatomical results, especially in moderate DME subgroups. The finding might be due to the multiplesided inhibiting mechanism of aflibercept. Further real-world experience reports are needed for comprehensive evaluation of our conclusions. 


\section{Acknowledgements}

None of the authors have any conflict of interest to disclose regarding this report. No financial support was obtained from any source for this research.

\section{Ethics}

Ethics Committee Approval: The ethics commitee approval was obtained from the local ethical commitee at istanbul Okmeydanı Training and Research Hospital (approval number: 1116).

Informed Consent: Written informed consent was obtained from each participicant of this study.

Peer-review: Externally peer-reviewed.

\section{Authorship Contributions}

Surgical and Medical Practices: B.E., A.C.., A.C.A., S.B., Concept: B.E., A.C.., S.B., Design: B.E., A.C.., M.N.E., Data Collection or Processing: A.C.A., B.E., S.B., A.C.., Analysis or Interpretation: B.E., A.Ç., Literature Search: B.E., A.Ç., A.C.A., S.B., Writing: B.E., A.C.., S.B.

Conflict of Interest: The authors of this study do not have any conflict of interest

Financial Disclosure: No financial support was received for this study.

\section{REFERENCES}

1. O'Doherty M, Dooley I, Hickey-Dwyer M. Interventions for diabetic macular oedema: a systematic review of the literature. $\mathrm{Br} J$ Ophthalmol 2008;92:1581-90.

2. Yau JW, Rogers SL, Kawasaki R, Lamoureux EL, Kowalski JW, Bek T, et al. Meta-Analysis for Eye Disease (META-EYE) Study Group. Global prevalence and major risk factors of diabetic retinopathy. Diabetes Care 2012;35:55664.

3. Diabetic Retinopathy Clinical Research Network, Wells JA, Glassman AR, Ayala AR, Jampol LM, Aiello LP, et al. Aflibercept, bevacizumab, or ranibizumab for diabetic macular edema. N Engl J Med 2015;372:1193-203.

4. Wells JA, Glassman AR, Ayala AR, Jampol LM, Bressler NM, et al. Aflibercept, Bevacizumab, or Ranibizumab for Diabetic Macular Edema: Two-Year Results from a Comparative Effectiveness Randomized Clinical Trial. Ophthalmology 2016;123:1351-9.

5. Haritoglou C, Kook D, Neubauer A, Wolf A, Priglinger S, Strauss R, et al Intravitreal bevacizumab (Avastin) therapy for persistent diffuse diabetic macular edema. Retina 2006;26:999-1005.

6. Nguyen QD, Brown DM, Marcus DM, Boyer DS, Patel S, Feiner L, et al Ranibizumab for diabetic macular edema: results from 2 phase III randomized trials: RISE and RIDE. Ophthalmology 2012;119:789-801.

7. Do DV, Nguyen QD, Boyer D, Schmidt-Erfurth U, Brown DM, Vitti R, et al. Oneyear outcomes of the da Vinci Study of VEGF Trap-Eye in eyes with diabetic macular edema. Ophthalmology 2012;119:1658-65.

8. Mitchell P, Bandello F, Schmidt-Erfurth U, Lang GE, Massin P, Schlingemann RO, et al. The RESTORE study: ranibizumab monotherapy or combined with laser versus laser monotherapy for diabetic macular edema. Ophthalmology 2011;118:615-25.

9. Boyer DS, Yoon YH, Belfort R Jr, Bandello F, Maturi RK, Augustin AJ, et al. Three-year, randomized, sham-controlled trial of dexamethasone intravitreal implant in patients with diabetic macular edema. Ophthalmology 2014;121:1904-14

10. Schmidt-Erfurth U, Garcia-Arumi J, Bandello F, Berg K, Chakravarthy U, Gerendas BS, et al. Guidelines for the Management of Diabetic Macular Edema by the European Society of Retina Specialists (EURETINA). Ophthalmologica 2017;237:185-222

11. Holash J, Davis S, Papadopoulos N, Croll SD, Ho L, Russell M, et al. VEGFTrap: a VEGF blocker with potent antitumor effects. Proc Natl Acad Sci U S A 2002;99:11393-8.

12. Stewart MW, Rosenfeld PJ. Predicted biological activity of intravitreal VEGF Trap. Br J Ophthalmol 2008;92:667-8.

13. Shimizu N, Oshitari T, Tatsumi T, Takatsuna Y, Arai M, Sato E, et al Comparisons of Efficacy of Intravitreal Aflibercept and Ranibizumab in Eyes with Diabetic Macular Edema. Biomed Res Int 2017;2017:1747108.

14. Brown DM, Schmidt-Erfurth U, Do DV, Holz FG, Boyer DS, Midena E, et al. Intravitreal Aflibercept for Diabetic Macular Edema: 100-Week Results From the VISTA and VIVID Studies. Ophthalmology 2015;122:2044-52.

15. Ishibashi T, Li X, Koh A, Lai TY, Lee FL, Lee WK, et al. The REVEAL Study: Ranibizumab Monotherapy or Combined with Laser versus Laser Monotherapy in Asian Patients with Diabetic Macular Edema. Ophthalmology 2015; 122:1402-15

16. Menchini U, Bandello F, De Angelis V, Ricci F, Bonavia L, Viola F, et al Ranibizumab for Visual Impairment due to Diabetic Macular Edema: Real-World Evidence in the Italian Population (PRIDE Study). J Ophthalmol 2015;2015:324841. 